\title{
A Spatio-Temporal Hybrid Strauss Hardcore Point Process for Forest Fire Occurrences
}

Morteza Raeisi ( $\nabla$ morteza.raeisi@univ-avignon.fr)

Avignon University https://orcid.org/0000-0002-9999-4097

Florent Bonneu

Avignon University

Edith Gabriel

INRAE, BioSP

\section{Research Article}

Keywords: Spatio-temporal Gibbs point processes, Hybridization, Multi-scale point processes, Forest fires

Posted Date: July 30th, 2021

DOI: https://doi.org/10.21203/rs.3.rs-711699/v1

License: (1) This work is licensed under a Creative Commons Attribution 4.0 International License.

Read Full License 


\title{
A spatio-temporal hybrid Strauss hardcore point process for forest fire occurrences
}

\author{
Morteza Raeisi • Florent Bonneu • \\ Edith Gabriel
}

Received: date / Accepted: date

\begin{abstract}
We propose a new point process model that combines, in the spatiotemporal setting, both multi-scaling by hybridization and hardcore distances. Our so-called hybrid Strauss hardcore point process model allows different types of interaction, at different spatial and/or temporal scales, that might be of interest in environmental and biological applications. The inference and simulation of the model are implemented using the logistic likelihood approach and the birth-death Metropolis-Hastings algorithm. Our model is used to describe forest fire occurrences in Spain.
\end{abstract}

Keywords Spatio-temporal Gibbs point processes · Hybridization · Multiscale point processes $\cdot$ Forest fires

\section{Introduction}

In point process modeling, most of existing models yield point patterns with mainly single-structure, but only a few with multi-structure. Interactions with single-structure are often classified into three classes: randomness, clustering and inhibition. Among inhibition processes is the hardcore process. It has some hardcore distance $h$ in which distinct points are not allowed to come closer than a distance $h$ apart. This type of interaction can be modelled by Gibbs point processes as the hardcore or Strauss hardcore point processes and also

M. Raeisi

LMA EA2151, Avignon University, F-84000 Avignon, France

Tel.: +33490843541

E-mail: morteza.raeisi@univ-avignon.fr

F. Bonneu

LMA EA2151, Avignon University, F-84000 Avignon, France

INRAE, BioSP, F-84914 Avignon, France

E. Gabriel

INRAE, BioSP, F-84914 Avignon, France 
by Cox point processes as Matérn's hardcore (Matérn, 1960; 1986) or Matérn thinned Cox point processes (Andersen and Hahn, 2016). Here, we focus on the former, i.e. Gibbs models implemented by a hardcore component as in the Strauss hardcore model. The form of Strauss hardcore density indicates that the hardcore parameter only rules at least distance between points, and has no effect on the interaction terms of the density (Dereudre and Lavancier, 2017, sect. 2.3).

In several domains, there exist point patterns with hardcore distances that have to be modelled. Spatial point patterns with hardcore property can be found in capillaries studies (Mattfeldt et al., 2006; 2007; 2009), in texture synthesis (Hurtut et al., 2009), in forest fires (Turner, 2009), in cellular networks (Taylor et al., 2012 and Ying et al., 2014), in landslides (Das and Stein, 2016), in modern and contemporary architecture and art (Stoyan, 2016) and in location clustering econometrics (Sweeney and Gomez-Antonio, 2016).

There also exist point patterns with either clustering and inhibition like hardcore interactions at different scales simultaneously (Badreldin et al., 2015; Andersen and Hahn, 2016 and Wang et al., 2020). Wang et al. (2020, sect. 2.4) investigated effect of the hardcore distance on spatial patterns of trees by comparing the pair correlation function curves for different values of hardcore distances in the fitted hybrid Geyer hardcore model. Raeisi et al. (2019) review spatial and spatio-temporal point processes that model both inhibition and clustering at different scales. Such multi-structure interactions can be modelled by the spatial hybrid Gibbs point process (Baddeley et al, 2013). In this paper, we aim to extend the spatial Strauss hardcore point process (Ripley, 1988) to the spatio-temporal framework and introduce a multi-scale version of it using hybridization approach. We use this model to describe one of the most complex phenomena from the spatio-temporal modeling point of view: forest fire occurrences.

The complexity of forest fire occurrences is due in particular to the existence of multi-scale structures and hardcore distances in space and time. For instance, spatio-temporal variations of fire occurrences depend on the spatial distribution of current land use and weather conditions. Changes in vegetation due to forest fires burnt areas further affect the probability of fire occurrences during the regeneration period leading to the existence of hardcore distances in space-time. The multi-scale structure of clustering and inhibition in the spatiotemporal pattern of the forest fire occurrences is discussed in Gabriel et al. (2017). Wildfires have mainly been modelled by Cox processes and inferred by Bayesian hierarchical approaches, as the integrated nested Laplace approximation (INLA) approach (Rue et al., 2009). See Møller and Diaz-Avalos (2010), Pereira et al. (2013), Serra et al. (2012, 2014a,b), Najafabadi et al. (2015), Juan (2020) and Pimont et al. (2021) for single-structure models and Gabriel et al. (2017), Opitz et al. (2020) for multi-structure models. Recently, Raeisi et al. (2021) modelled the multi-structure of forest fire occurrences by a spatiotemporal Gibbs process and use a composite likelihood approach for its inference. 
This paper is organized as follows. In Section 2 we introduce in the spatiotemporal framework the notations and definitions of Gibbs point processes in order to introduce our multi-scale version of the Strauss hardcore model. Section 3 is devoted to the inference of our model. It describes techniques to determine the irregular parameters (hardcore and interaction distances) and the logistic-likelihood approach generalized to the spatio-temporal setting to estimate the regular parameters (strength of interactions). Section 4 illustrates the goodness-of-fit of the logistic likelihood approach on simulated patterns of our model obtained by an extended Metropolis-Hastings algorithm. Finally in Section 5, we apply our model to monthly records of forest fires in the center of Spain.

\section{Towards multi-scale Strauss hardcore point processes}

Gibbs models are flexible point processes that allow the specification of point interactions via a probability density defined with respect to the unit rate Poisson point process. These models allow to characterize a form of local or Markovian dependence amongst events. Gibbs point processes contain a large class of flexible and natural models that can be applied for:

- Postulating the interaction mechanisms between pairs of points,

- Taking into account clustering, randomness or inhibition structures,

- Combining several structures at different scales with the hybridization approach.

Let $\mathbf{x}=\left\{\left(\xi_{1}, t_{1}\right), \ldots,\left(\xi_{n}, t_{n}\right)\right\}$ be a spatio-temporal point pattern where $\left(\xi_{i}, t_{i}\right) \in W=S \times T \subset \mathbb{R}^{2} \times \mathbb{R}$. We consider $(W, d(\cdot, \cdot))$ where $d\left((u, v),\left(u^{\prime}, v^{\prime}\right)\right):=$ $\max \left\{\left\|u-u^{\prime}\right\|,\left|v-v^{\prime}\right|\right\}$ for $(u, v),\left(u^{\prime}, v^{\prime}\right) \in W$ is a complete, separable metric space. The cylindrical neighbourhood $C_{r}^{q}(u, v)$ centred at $(u, v) \in W$ is defined by

$$
C_{r}^{q}(u, v)=\{(a, b) \in W:|| u-a \| \leq r,|v-b| \leq q\},
$$

where $r, q>0$ are spatial and temporal radius and $\|\cdot\|$ denotes the Euclidean distance in $\mathbb{R}^{2}$ and $|\cdot|$ denotes the usual distance in $\mathbb{R}$. Note that $C_{r}^{q}(u, v)$ is a cylinder with centre $(u, v)$, radius $r$, and height $2 q$.

A finite Gibbs point process is a finite simple point process defined with a density $f(\mathbf{x})$ that satisfies the hereditary condition, i.e. $f(\mathbf{x})>0 \Rightarrow f(\mathbf{y})>0$ for all $\mathbf{y} \subset \mathbf{x}$.

A closely related concept to density functions is Papangelou conditional intensity function (Papangelou, 1974) which is a tool for simulating Gibbs models and inferring its parameters. The Papangelou conditional intensity of a spatio-temporal point process on $W$ with density $f$ is defined, for $(u, v) \in W$, by

$$
\lambda((u, v) \mid \mathbf{x})=\frac{f(\mathbf{x} \bigcup(u, v))}{f(\mathbf{x} \backslash(u, v))},
$$

with $a / 0:=0$ for all $a \geq 0$ (Cronie and van Lieshout, 2015). 
The Papangelou conditional intensity is also very useful to describe local interactions in a point pattern, and leads to the notion of a Markov point process which is the basis for the implementation of MCMC algorithms used for simulating of Gibbs models. We say that the point process has "interactions of range $R$ at $(\xi, t)$ " if points further than $R$ away from $(\xi, t)$ do not contribute to the conditional intensity at $(\xi, t)$. A spatio-temporal Gibbs point process $X$ has a finite interaction range $R$ if the Papangelou conditional intensity satisfies

$$
\lambda((u, v) \mid \mathbf{x})=\lambda\left((u, v) \mid \mathbf{x} \cap C_{R}^{R}(u, v)\right)
$$

for all configurations $\mathbf{x}$ of $X$ and all $(u, v) \in W$, where $C_{R}^{R}(u, v)$ denotes the cylinder of radius $R>0$ and height $2 R>0$ centred at $(u, v)$. Spatio-temporal Gibbs models usually have finite interaction range property (spatio-temporal Markov property) and are called in this case Markov point processes (van Lieshout 2000). The finite range property of a spatio-temporal Gibbs model implies that the probability to insert a point $(u, v)$ into $\mathbf{x}$ depends only on some cylindrical neighborhood of $(u, v)$.

Here, we first review spatio-temporal Gibbs models and then extend the spatial Strauss hardcore model to the spatio-temporal and multi-scale context. We further refer to Dereudre (2019) for more formal introduction of Gibbs point processes.

\subsection{Single-scale Gibbs point process models}

In the literature, several spatio-temporal Gibbs point process models have been proposed such as the hardcore (Cronie and van Lieshout, 2015), Strauss (Gonzalez et al., 2016), area-interaction (Iftimi et al., 2018), and Geyer (Raeisi et al., 2021) point processes.

A Gibbs point process model explicitly postulates that interactions traduce dependencies between the points of the pattern. The hardcore interaction is one of the simplest type of interaction, which forbids points being too close to each other. The homogeneous spatio-temporal hardcore point process is defined by the density

$$
f(\mathbf{x})=c \lambda^{n(\mathbf{x})} \mathbb{1}\left\{\left\|\xi-\xi^{\prime}\right\|>h_{s} \text { or }\left|t-t^{\prime}\right|>h_{t} ; \forall(\xi, t) \neq\left(\xi^{\prime}, t^{\prime}\right) \in \mathbf{x}\right\},
$$

with respect to a unit rate Poisson point process on $W$, where $c>0$ is a normalizing constant, $\lambda>0$ is an activity parameter, $h_{s}, h_{t}>0$ are, respectively, the spatial and the temporal hardcore distances and $n(\mathbf{x})$ is the number of points in $\mathbf{x}$. The Papangelou conditional intensity of a homogeneous spatiotemporal hardcore point process for $(u, v) \notin \mathbf{x}$ is obtained

$$
\begin{aligned}
\lambda((u, v) \mid \mathbf{x}) & =\lambda \mathbb{1}\left\{\|\xi-u\|>h_{s} \text { or }|t-v|>h_{t} ; \forall(\xi, t) \in \mathbf{x}\right\} \\
& =\lambda \prod_{(\xi, t) \in \mathbf{x}} \mathbb{1}\left\{|| \xi-u \|>h_{s} \text { or }|t-v|>h_{t}\right\} \\
& =\lambda \prod_{(\xi, t) \in \mathbf{x}} \mathbb{1}\left\{(\xi, t) \notin C_{h_{s}}^{h_{t}}(u, v)\right\} .
\end{aligned}
$$


. The homogeneous spatio-temporal Strauss point process is defined by density

$$
f(\mathbf{x})=c \lambda^{n(\mathbf{x})} \gamma^{S_{r}^{q}(\mathbf{x})}
$$

with respect to a unit rate Poisson point process on $W$, where

$$
S_{r}^{q}(\mathbf{x})=\sum_{(\xi, t) \neq\left(\xi^{\prime}, t^{\prime}\right) \in \mathbf{x}} \mathbb{1}\left\{|| \xi-\xi^{\prime} \| \leq r,\left|t-t^{\prime}\right| \leq q\right\}
$$

and the Papangelou conditional intensity of the model is

$$
\text { for }(u, v) \notin \mathbf{x}, \quad \lambda((u, v) \mid \mathbf{x})=\lambda \gamma^{n\left[C_{r}^{q}(u, v) ; \mathbf{x}\right]},
$$

and

$$
\text { for }(\xi, t) \in \mathbf{x}, \quad \lambda((\xi, t) \mid \mathbf{x})=\lambda \gamma^{n\left[C_{r}^{q}(\xi, t) ; \mathbf{x} \backslash(\xi, t)\right]},
$$

where $n\left[C_{r}^{q}(y, z) ; \mathbf{x}\right]=\sum_{(\xi, t) \in \mathbf{x}} \mathbb{1}\{\|y-\xi\| \leq r,|z-t| \leq q\}$ is the number of points in $\mathbf{x}$ which are in a cylinder $C_{r}^{q}(y, z)$. Although the Strauss point process was originally intended as a model of clustering, it can only be used to model inhibition, because the parameter $\gamma$ cannot be greater than 1 . If we take $\gamma>1$, the density function of Strauss model is not integrable, so it does not define a valid probability density.

As mentioned, the Strauss point process model only achieves the inhibition structure. In the spatial framework, two ways are introduced to overcome this problem that we extend to the spatio-temporal framework hence defining two new spatio-temporal Gibbs point process models.

A first way is to consider an upper bound for the number of neighboring points that interact. In this case, Raeisi et al. (2021) defined a homogeneous spatio-temporal Geyer saturation point process by density

$$
f(\mathbf{x})=c \lambda^{n(\mathbf{x})} \prod_{(\xi, t) \in \mathbf{x}} \gamma^{\min \left\{s, n^{*}\left[C_{r}^{q}(\xi, t) ; \mathbf{x}\right]\right\}},
$$

with respect to a unit rate Poisson point process on $W$, where $s$ is a saturation parameter and $n^{*}\left[C_{r}^{q}(\xi, t) ; \mathbf{x}\right]=n\left[C_{r}^{q}(\xi, t) ; \mathbf{x} \backslash(\xi, t)\right]=\sum_{(u, v) \in \mathbf{x} \backslash(\xi, t)} \mathbb{1}\{\| u-$ $\xi \| \leq r,|v-t| \leq q\}$.

A second way is to introduce a hardcore condition to the Strauss density (6). Hence, we can define a Strauss hardcore model in the spatio-temporal context.

Definition 1 We define the spatio-temporal Strauss hardcore point process as the point process with density

$$
f(\mathbf{x})=c \lambda^{n(\mathbf{x})} \gamma^{S_{r}^{q}(\mathbf{x})} \mathbb{1}\left\{\left\|\xi-\xi^{\prime}\right\|>h_{s} \text { or }\left|t-t^{\prime}\right|>h_{t} ; \forall(\xi, t) \neq\left(\xi^{\prime}, t^{\prime}\right) \in \mathbf{x}\right\},
$$

where $0<h_{s}<r$ and $0<h_{t}<q$. 
The model could be used to model clustering patterns with a softer attraction between the points like a pattern with a combination of interaction terms that show repulsion between the points at a small scale and attraction between the points at a larger scale. The Papangelou conditional intensity of a homogeneous spatio-temporal Strauss hardcore point process for $(u, v) \notin \mathbf{x}$ is obtained

$$
\begin{aligned}
\lambda((u, v) \mid \mathbf{x}) & =\lambda \gamma^{n\left[C_{r}^{q}(u, v) ; \mathbf{x}\right]} \mathbb{1}\left\{\|\xi-u\|>h_{s} \text { or }|t-v|>h_{t} ; \forall(\xi, t) \in \mathbf{x}\right\} \\
& =\lambda \gamma^{n\left[C_{r}^{q}(u, v) ; \mathbf{x}\right]} \prod_{(\xi, t) \in \mathbf{x}} \mathbb{1}\left\{(\xi, t) \notin C_{h_{s}}^{h_{t}}(u, v)\right\} .
\end{aligned}
$$

We can define inhomogeneous versions of all above models by replacing the constant $\lambda$ by a function $\lambda(\xi, t)$, inside the product operator over $(\xi, t) \in$ $\mathbf{x}$, that expresses a spatio-temporal trend, which can be a function of the coordinates of the points and depends on covariate information.

\subsection{Multi-scale Gibbs point process models}

Since most natural phenomena exhibit dependence at multiple scales as earthquake (Siino et al., 2017;2018) and forest fire occurrences (Gabriel et al., 2017), single-scale Gibbs point process models are unrealistic in many applications. This motivates us and other statisticians to construct multi-scale generalizations of the classical Gibbs models. Baddeley et al. (2013) proposed hybrid models as a general way to generate multi-scale processes combining Gibbs processes. Given $m$ densities $f_{1}, f_{2}, \ldots, f_{m}$ of Gibbs point processes, the hybrid density is defined as $f(\mathbf{x})=c f_{1}(\mathbf{x}) \times f_{2}(\mathbf{x}) \times \cdots \times f_{m}(\mathbf{x})$ where $c$ is a normalization constant.

Iftimi et al. (2018) extended the hybrid approach for an area-interaction model to the spatio-temporal framework where the density is given by

$$
f(\mathbf{x})=c \prod_{(\xi, t) \in \mathbf{x}} \lambda(\xi, t) \prod_{j=1}^{m} \gamma_{j}^{-\ell\left(\cup_{(\xi, t) \in \mathbf{x}} C_{r_{j}}^{q_{j}}(\xi, t)\right)},
$$

with respect to a unit rate Poisson process on $W$, where $\left(r_{j}, q_{j}\right)$ are pairs of irregular parameters of the model and $\gamma_{j}$ are interaction parameters, $j=$ $1, \ldots, m$.

In the same way, Raeisi et al. (2021) defined a spatio-temporal multi-scale Geyer saturation point process with density

$$
f(\mathbf{x})=c \prod_{(\xi, t) \in \mathbf{x}} \lambda(\xi, t) \prod_{j=1}^{m} \gamma_{j}^{\min \left\{s_{j}, n\left(C_{r_{j}}^{q_{j}}(\xi, t) ; \mathbf{x}\right)\right\}}
$$

with respect to a unit rate Poisson process on $W$, where $c>0$ is a normalizing constant, $\lambda \geq 0$ is a measurable and bounded function, $\gamma_{j}>0$ are the interaction parameters.

Similarly, a hybrid version of spatio-temporal Strauss model can be defined by hybridization. 
Definition 2 We define the spatio-temporal hybrid Strauss point process with density

$$
f(\mathbf{x})=c \prod_{(\xi, t) \in \mathbf{x}} \lambda(\xi, t) \prod_{j=1}^{m} \gamma_{j}^{S_{r_{j}}^{q_{j}}(\mathbf{x})},
$$

with respect to a unit rate Poisson process on $W$.

Note that we called the model (14) hybrid rather than multi-scale. The model (14) can cover inhibition structure because $0<\gamma_{j}<1, \forall j \in\{1, \ldots, m\}$. However, it can take into account clustering if one of densities in hybrid is the one of a hardcore process.

\subsection{Hybrid Strauss hardcore point process}

The hybrid Gibbs point process models do not necessarily include $m$ same Gibbs point process models (see Baddeley et al., 2015 sect. 13.8). Badreldin et al. (2015) applied a spatial hybrid model including a hardcore density to model strong inhibition at very short distances, Geyer density for cluster structure in short to medium distances and a Strauss density for a randomness structure in larger distances to the spatial pattern of the halophytic species distribution in an arid coastal environment. Wang et al. (2020) fitted a spatial hybrid Geyer hardcore point process on the tree spatial distribution patterns. In this section, we extend this type of hybrids to the spatio-temporal context.

Definition 3 We define the spatio-temporal hybrid Strauss hardcore point process with density

$$
\begin{aligned}
f(\mathbf{x})= & c \prod_{(\xi, t) \in \mathbf{x}} \lambda(\xi, t) \prod_{j=1}^{m} \gamma_{j}^{S_{r_{j}}^{q_{j}}(\mathbf{x})} \\
& \times \mathbb{1}\left\{\left\|\xi^{\prime}-\xi^{\prime \prime}\right\|>h_{s} \text { or }\left|t^{\prime}-t^{\prime \prime}\right|>h_{t} ; \forall\left(\xi^{\prime}, t^{\prime}\right) \neq\left(\xi^{\prime \prime}, t^{\prime \prime}\right) \in \mathbf{x}\right\},
\end{aligned}
$$

where $0<h_{s}<r_{1}<\cdots<r_{m}$ and $0<h_{t}<q_{1}<\cdots<q_{m}$.

The Papangelou conditional intensity of an inhomogeneous spatio-temporal hybrid Strauss hardcore process is then, for $(u, v) \notin \mathbf{x}$,

$$
\begin{aligned}
\lambda((u, v) \mid \mathbf{x}) & =\lambda(u, v) \prod_{j=1}^{m} \gamma_{j}^{n\left[C_{r_{j}}^{q_{j}}(u, v) ; \mathbf{x}\right]} \mathbb{1}\left\{\|\xi-u\|>h_{s} \text { or }|t-v|>h_{t} ; \forall(\xi, t) \in \mathbf{x}\right\} \\
& =\lambda(u, v) \prod_{j=1}^{m} \gamma_{j}^{n\left[C_{r_{j}}^{q_{j}}(u, v) ; \mathbf{x}\right]} \prod_{(\xi, t) \in \mathbf{x}} \mathbb{1}\left\{(\xi, t) \notin C_{h_{s}}^{h_{t}}(u, v)\right\} .
\end{aligned}
$$

Because, the conditional intensity of Gibbs models including a hardcore interaction term takes the value zero at some locations, we can rewrite it as

$$
\lambda((u, v) \mid \mathbf{x})=m((u, v) \mid \mathbf{x}) \lambda^{+}((u, v) \mid \mathbf{x}),
$$


where $m((u, v) \mid \mathbf{x})$ takes only the values 0 and 1 , and $\lambda^{+}((u, v) \mid \mathbf{x})>0$ everywhere.

The spatio-temporal hybrid Strauss hardcore point process (15) is a Markov point process in Ripley-Kelly's (1977) sense at interaction range $\max \left\{r_{m}, q_{m}\right\}$. This can be shown as in Iftimi et al. (2018) and Raeisi et al. (2021).

\section{Inference}

Gibbs point process models involve two types of parameters: regular and irregular parameters. A parameter is called regular if the log likelihood of density is a linear function of that parameter otherwise it is called irregular. Typically, regular parameters determine the 'strength' of the interaction, while irregular parameters determine the 'range' of the interaction. As an example, in the Strauss hardcore point process (10), the trend parameter $\lambda$ and the interaction $\gamma$ are regular parameters and the interaction distances $r$ and $q$ and the hardcore distances $h_{s}$ and $h_{t}$ are irregular parameters.

To determine the interaction distances $r$ and $q$, there are several practical techniques, but no general statistical theory available. A useful technique is the maximum profile pseudo-likelihood approach (Baddeley and Turner, 2000). In the spatio-temporal framework, Iftimi et al. (2018) and Raeisi et al. (2021) selected feasible range of irregular parameters by analyzing the behavior of some summary statistics and the goodness-of-fit of several models with different combinations of parameters.

The hardcore interaction term $m(\cdot \mid \mathbf{x})$ in the conditional intensity (17) does not depend on the other parameters of the densities of Gibbs point processes. This implies that it can first be estimated and kept fixed for the sequel (Baddeley et al., 2019, p. 26). In the spatial framework, the maximum likelihood estimate of the hardcore distance in $m(\cdot \mid \mathbf{x})$ corresponds to the minimum interpoint distance (Baddeley et al., 2013, Lemma 7). The generalization to the spatio-temporal context with a cylindrical hardcore structure implies to consider a multi-objective minimization problem over the spatial and temporal hardcore distances $h_{s}$ and $h_{t}$. The choice of our hardcore parameters needs to analyze the Pareto front of feasible solutions on the graph of spatial and temporal interpoint distances. We refer the reader to Ehrgott (2005) for a description of multi-criteria optimization and the definition of Pareto optimality. To estimate the hardcore distance $h_{s}$ and $h_{t}$, we consider a feasible solution on the Pareto front as large as possible and with a ratio consistent with our knowledge of interaction mechanisms in practice.

Regular parameters can be estimated using the pseudo-likelihood method (Baddeley and Turner, 2000) or logistic likelihood method (Baddeley et al., 2014) rather than the maximum likelihood method (Ogata and Tanemura, 1981). Due to the advantage of the logistic likelihood over pseudo-likelihood for spatio-temporal Gibbs point processes (Iftimi et al., 2018; Raeisi et al., 2021), we implement the former approach in Raeisi et al. (2021, Algorithm 
2) for regular parameter estimation of the spatio-temporal hybrid Strauss hardcore point process.

We assume that $\boldsymbol{\theta}=\left(\log \gamma_{1}, \log \gamma_{2}, \ldots, \log \gamma_{m}\right)$ is the logarithm of interaction parameters in spatio-temporal hybrid Strauss hardcore point process (15). To estimate $\boldsymbol{\theta}$, due to (17), we just consider the points $(u, v)$ where $m((u, v) \mid \mathbf{x})$ is equal to 1 in (16). By defining $S_{j}((u, v), \mathbf{x}):=n\left[C_{r_{j}}^{q_{j}}(u, v) ; \mathbf{x} \backslash(u, v)\right]$ in (16), we can thus write $\lambda_{\boldsymbol{\theta}}((u, v) \mid \mathbf{x})=\lambda(u, v) \prod_{j=1}^{m} \exp \left(\theta_{j} S_{j}((u, v), \mathbf{x})\right) .=$ Hence, the logarithm of the Papangelou conditional intensity of the spatio-temporal hybrid Strauss hardcore point process for $(u, v) \in W$ which satisfies in hardcore condition, i.e. $m((u, v) \mid \mathbf{x})=1$ in $(16)$, is

$$
\begin{aligned}
\log \lambda((u, v) \mid \mathbf{x}) & =\log \lambda(u, v)+\sum_{j=1}^{m}\left(\log \gamma_{j}\right) S_{j}((u, v), \mathbf{x}) \\
& =\log \lambda(u, v)+\boldsymbol{\theta}^{\top} \boldsymbol{S}((u, v), \mathbf{x})
\end{aligned}
$$

corresponding to a linear model in $\boldsymbol{\theta}$ with offset $\log \lambda(u, v)$ where $\boldsymbol{S}((u, v), \mathbf{x})=$ $\left[S_{1}((u, v), \mathbf{x}), S_{2}((u, v), \mathbf{x}), \ldots, S_{m}((u, v), \mathbf{x})\right]^{\top}$ is a sufficient statistics.

By considering a set of dummy points $\mathbf{d}$ from an independent Poisson process with intensity function $\rho$, we obtain by defining the Bernoulli variables $Y((\xi, t))=\mathbb{1}_{\{(\xi, t) \in \mathbf{x}\}}$ for $(\xi, t) \in \mathbf{x} \cup \boldsymbol{d}$ that the logit of $P(Y((\xi, t)))=1$ is equal to $\log \frac{\lambda_{\boldsymbol{\theta}}((\xi, t) \mid \mathbf{X} \backslash(\xi, t))}{\rho(\xi, t)}$. Under regularity conditions, the log-logistic likelihood

$$
\begin{aligned}
\log L L(\mathbf{x}, \boldsymbol{d} ; \boldsymbol{\theta}) & =\sum_{(\xi, t) \in \mathbf{x}} \log \frac{\lambda_{\boldsymbol{\theta}}((\xi, t) \mid \mathbf{x})}{\lambda_{\boldsymbol{\theta}}((\xi, t) \mid \mathbf{x})+\rho(\xi, t)} \\
& +\sum_{(\xi, t) \in \boldsymbol{d}} \log \frac{\rho(\xi, t)}{\lambda_{\boldsymbol{\theta}}((\xi, t) \mid \mathbf{x})+\rho(\xi, t)},
\end{aligned}
$$

admits a unique maximum. By consequence, the estimation of $\boldsymbol{\theta}$ in the Papangelou conditional intensity is equivalent to the estimation of logistic regression parameters, already implemented by using standard software for GLMs. The logistic regression

$$
\log \frac{\lambda_{\boldsymbol{\theta}}((\xi, t) \mid \mathbf{x})}{\rho(\xi, t)}=\log \frac{\lambda(\xi, t)}{\rho(\xi, t)}+\sum_{j=1}^{m} \theta_{j} S_{j}((\xi, t), \mathbf{x})
$$

is a linear model in $\boldsymbol{\theta}$ with offset $\log \frac{\lambda(\xi, t)}{\rho(\xi, t)}$. We use the approach of Raeisi et al. (2021) for data and dummy points such that $m(\cdot \mid \mathbf{x})=1$. We also consider that $\lambda(\xi, t)=\beta \mu(\xi, t)$, where $\mu(\xi, t)$ is a trend preliminary estimated with spatio-temporal covariates.

\section{Simulation study}

Due to the markovian property of the spatio-temporal hybrid Strauss hardcore point process (15), its Papangelou conditional intensity at a point thus depends 
Table 1 Parameter combinations of three hybrid Strauss hardcore point process models used in simulation study.

\begin{tabular}{cccccc}
\hline & \multicolumn{4}{c}{ Values of parameter } \\
\cline { 2 - 3 } \cline { 5 - 6 } Model & $\lambda$ & $\gamma$ & & Irregular parameters \\
\cline { 2 - 3 } \cline { 5 - 5 } Model 1 & 70 & $(0.8, .08)$ & & $(0.05,0.1)$ & $(0.01,0.01)$ \\
Model 2 & 50 & $(1.5,1.5)$ & & $(0.05,0.1)$ & $(0.01,0.01)$ \\
Model 3 & 70 & $(0.5,1.5)$ & & $(0.05,0.1)$ & $(0.01,0.01)$ \\
\hline
\end{tabular}

Table 2 Mean and 95\% interval regular parameter estimates of the three hybrid Strauss hardcore point process models used in simulation study.

\begin{tabular}{ccc}
\hline True values & Mean & $95 \%$ CI \\
\hline & \multicolumn{2}{c}{ Model 1 } \\
\cline { 2 - 3 }$\lambda=70$ & 71.43 & $(69.16,73.70)$ \\
$\gamma_{1}=0.8$ & 0.89 & $(0.78,1.00)$ \\
$\gamma_{2}=0.8$ & 0.78 & $(0.74,0.82)$ \\
\hline & \multicolumn{2}{c}{ Model 2 } \\
\cline { 2 - 3 }$\lambda=50$ & 50.84 & $(48.99,52.68)$ \\
$\gamma_{1}=1.5$ & 1.41 & $(1.23,1.60)$ \\
$\gamma_{2}=1.5$ & 1.46 & $(1.38,1.54)$ \\
\hline & \multicolumn{2}{c}{ Model 3 } \\
$\lambda=70$ & 71.67 & $(69.18,74.15)$ \\
$\gamma_{1}=0.5$ & 0.50 & $(0.43,0.57)$ \\
$\gamma_{2}=1.5$ & 1.49 & $(1.42,1.55)$ \\
\hline
\end{tabular}

only on that point and its neighbors in $\mathbf{x}$. Hence, We can design simulation approach by Markov chain Monte Carlo algorithms.

Gibbs point process models can be simulated a birth-death MetropolisHastings algorithm that typically requires only computation of the Papangelou conditional intensity (Møller and Waagepetersen, 2004). Raeisi et al. (2021) extended the birth-death Metropolis-Hastings algorithm to the spatiotemporal context that we adapt here for simulating the spatio-temporal hybrid Strauss hardcore point process.

We implement the estimation and simulation algorithms in $\mathrm{R}$ ( $\mathrm{R}$ Core Team, 2016) and generate simulations of three stationary spatio-temporal hybrid Strauss hardcore point processes specified by a conditional intensity of the form (16) in $W=[0,1]^{3}$. The parameter values used for the simulations are reported in Table 1. The spatial and temporal radii $r$ and $q$, spatial and temporal hardcores $h_{s}$ and $h_{t}$, are treated as known parameters.

We generate 100 simulations of each specified model. Boxplots of parameter estimates $\lambda, \gamma_{1}$, and $\gamma_{2}$ obtained from the logistic likelihood estimation method for each model are shown in Figure 1. The red horizontal lines represent the true parameter values. Point and interval parameter estimates $\lambda, \gamma_{1}$, and $\gamma_{2}$ are reported in Table 2. Most of the estimated parameter values are close to the true values for three models. Due to visual and computational comparisons, we conclude that the logistic likelihood approach performs well for spatiotemporal hybrid Strauss hardcore point processes. 

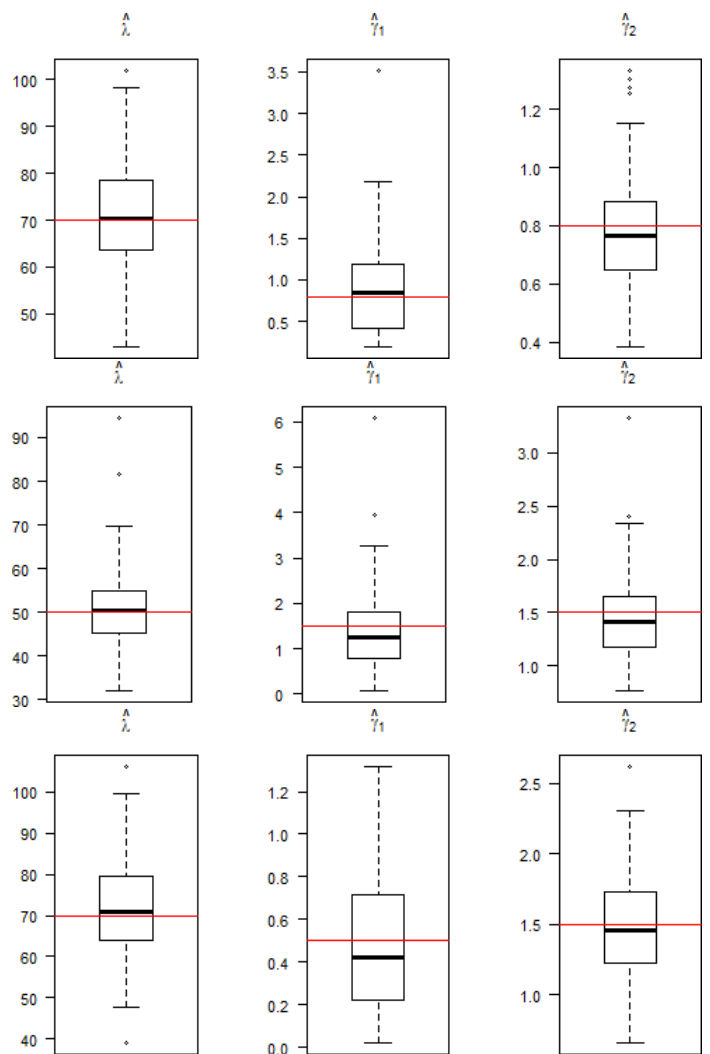

Fig. 1 Boxplots of regular parameter estimates of the hybrid Strauss hardcore point process obtained from the logistic likelihood estimation methods. Up to down: Model 1, Model 2, and Model 3

\section{Application}

In this section we aim to model the interactions of forest fire occurrences across a range of spatio-temporal scales.

\subsection{Data description}

The clmfires dataset available in spatstat package records the occurrences of forest fires in the region of Castilla-La Mancha, Spain (Figure 2, left) from 1998 to 2007 . The study area is approximately $400 \mathrm{~km} \times 400 \mathrm{~km}$. The clmfires dataset has already been used in some academic works devoted to the point process theory (see e.g. Juan et al., 2010; Gomez-Rubio, 2020, sect. 7.4.2; Myllymäki et al., 2020). The dataset has two levels of precision: from 1998 to 2003 locations were recorded as the centroids of the corresponding "district 


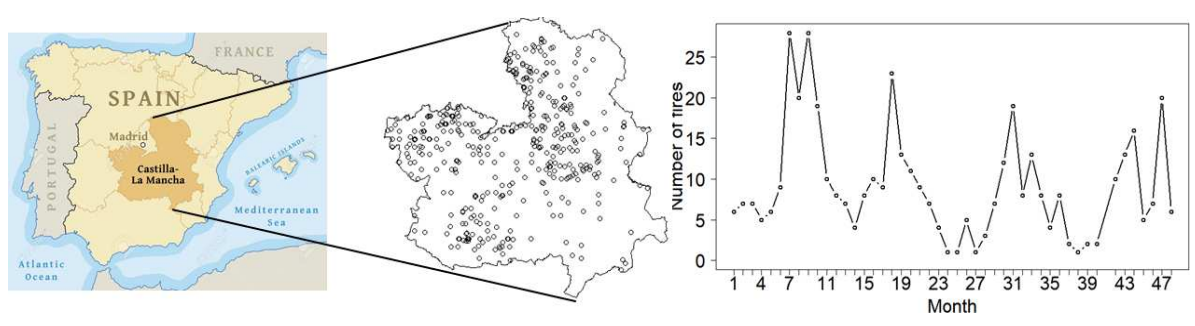

Fig. 2 Left: Map of region of Castilla-La Mancha (Spain). Middle: Forest fire locations. Right: monthly numbers of fires recorded between January 2004 and December 2007 with burnt areas, spatial distances and time distances respectively bigger than $5 \mathrm{ha}, 0.2 \mathrm{~km}$ and 100 days.

units", while since 2004 locations correspond to the exact UTM coordinates of the fire locations.

Due to the low precision of fire locations for the years 1998 to 2003 (GomezRubio 2020, sect. 7.4.2), we focus on fires in the period 2004 to 2007. In this period, we consider large forest fires with burnt areas larger than $5 \mathrm{ha}$. Figure 2 (middle) shows the point pattern of 432 wildfire locations onto the spatial region.

Due to memory constraints and availability of climate covariates in months, we consider monthly fire occurrences. The temporal component of the process takes integer values from 1 to 48. We thus consider $W=S \times T$ where $S$ is the region of Castilla-la-Mancha and $T=\{1,2, \ldots, 48\}$ corresponds to the months since January 2004. Figure 2 (right) shows the monthly number of fires occurring during our time period. We observe seasonal effects with notably large numbers of fires in summer that could be caused by high temperatures and low precipitations in this period and also by human activities.

In point pattern analysis, the spatial (spatio-temporal) inhomogeneity of patterns is notably driven by covariates. The clmfires dataset contains four environmental covariates that we include in our analysis: elevation, orientation, slope and land use. The covariates are known on a spatial grid with pixels of $4 \mathrm{~km} \times 4 \mathrm{~km}$, resulting in a total of 10,000 pixels. The land use is a factor-valued covariate whereas the others are real-valued covariates. We also consider weather data freely provided by the WorldClim database ${ }^{1}$ and containing monthly maximum temperatures $\left({ }^{\circ} \mathrm{C}\right)$ and total precipitations $(\mathrm{mm})$. Figure 3 illustrates the environmental covariates, which are considered fixed during our temporal period, and the climate covariates in January 2007.

\subsection{Estimation}

First, we estimate the trend function by considering a generalized linear model (GLM) on covariates. Then, by an exploratory analysis using spatio-temporal

\footnotetext{
1 https://www.worldclim.org
} 


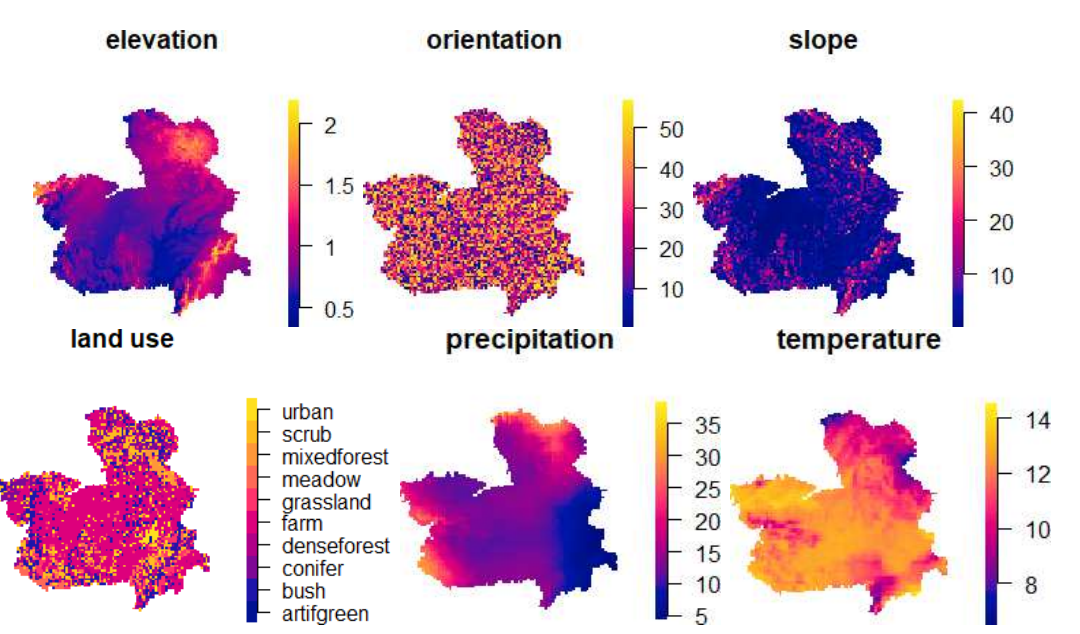

Fig. 3 Image plot of environmental covariates (elevation, orientation, slope and land use) and climate covariates (precipitation and temperature) in January 2007.

summary statistics we approximate the hardcore parameters and the interaction ranges. Finally, we use the logistic likelihood approach described in Section 3 for the estimation of regular parameters of our model with the trend function estimated in the preliminary step.

\subsubsection{Trend estimate}

Since covariates are available on a spatial grid, we restrict our attention on the related grid centers $\xi_{i}, i=1, \ldots, 10000$ and months $\left\{t_{j}\right\}_{j=1, \ldots, 48} \in T$ and consider $N_{i j} \mid \lambda\left(\xi_{i}, t_{j}\right) \sim$ Poisson $\left(\lambda\left(\xi_{i}, t_{j}\right)\right)$ where $N_{i j}$ is the number of forest fires in the $i^{\text {th }}$ grid center at month $t_{j}$.

Following Raeisi et al. (2021), by considering a GLM with Poisson response, we obtain:

$$
\log \lambda\left(\xi_{i}, t_{i}\right)=\beta_{0}+\sum_{k=1}^{6} \beta_{k} Z_{k}\left(\xi_{i}, t_{i}\right),
$$

where $Z_{k}\left(\xi_{i}, t_{i}\right), k=1, \ldots, 6$, are the environmental and climatic covariates at point $\left(\xi_{i}, t_{i}\right)$ and $\beta_{0}, \beta_{k}, k=1, \ldots, 6$ are the coefficients to estimate. As said before, we consider the same values for environmental covariates over time. A straightforward way to fit a GLM in R is to use the function glm. Table 3 reports the estimated coefficients in (21) and their significance level by a two-tailed Student's t-test. Coefficients higher (respectively lower) than zero imply an increase (resp. decrease) of the expected mean number of forest fires when the covariate value increase (resp. decrease). Those related to elevation and temperature are positively significant, showing that these two covariates favors the ignition of wildfires. At the opposite, the covariate precipitation has a negative significant coefficient indicating that an increase of the amount of 
Table 3 Estimated coefficients, standard errors and $p$-values based on two-tailed Student's t-tests of significant differences from zero.

\begin{tabular}{lccr}
\hline Coefficients & Estimate & Standard error & \multicolumn{2}{c}{$p$-value } \\
\hline$\beta_{0}$ (intercept) & -8.468 & 0.298 & $<2 \times 10^{-16}{ }^{* * *}$ \\
$\beta_{1}$ (elevation) & 0.546 & 0.164 & $0.001^{* * *}$ \\
$\beta_{2}$ (orientation) & 0.005 & 0.003 & 0.114 \\
$\beta_{3}$ (slope) & -0.019 & 0.01 & 0.054 \\
$\beta_{4}$ (land use) & -0.009 & 0.024 & 0.689 \\
$\beta_{5}$ (precipitation) & -0.007 & 0.002 & $0.003^{* *}$ \\
$\beta_{6}$ (temperature) & 0.054 & 0.006 & $<2 \times 10^{-16^{* *}}$
\end{tabular}

precipitation induces a decrease in the mean number of forest fires. The land use appears not significantly different from zero, it can be explained by the low spatial resolution of the covariates.

\subsubsection{Irregular parameter estimates}

We have two types of irregular parameters in our spatio-temporal Gibbs point process. On the one hand, the hardcore distances that we can choose among the feasible solutions on the Pareto front of spatial and temporal interpoint distances. According to Figure 4, we choose on the Pareto front the unique feasible solution in our case that gives non-zero values for the two hardcore distances, i.e. $h_{s}=0.35 \mathrm{~km}$ and $h_{t}=1$ month. On the other hand, for the nuisance parameters $m, r_{j}$ and $q_{j}, j=1, \ldots, m$, there is no common method for estimating them. Here we considered several combinations of ad-hoc values within a reasonable range and select the optimal irregular parameters according to the Akaike's Information Criterion (AIC) of the fitted model after the regular parameter estimation step (Raeisi et al., 2021). We chose 25 configurations of reasonable range for the nuisance parameters using a preliminary spatio-temporal exploratory analysis of the interaction ranges done with the inhomogeneous pair correlation function, the maximum nearest neighbor distance and the temporal auto-correlation function. We fitted the spatiotemporal hybrid Strauss point process model for a range of ad-hoc values $r_{j} \in(0.35,20], q_{j} \in\{2, \ldots, 15\}, j=1, \ldots, m$ and $m \in\{1, \ldots, 6\}$. The minimum AIC is obtained for the combination given in Table 4.

\subsubsection{Regular parameter estimates}

We consider the logistic likelihood method investigated in Section 3 to estimate the regular parameters. We simulate dummy points from an inhomogeneous Poisson point process with intensity $\rho(\xi, t)=C \hat{\lambda}(\xi, t) / \nu$ where $C=4$ by a classical rule of thumb in the logistic likelihood approach, $\hat{\lambda}$ is the estimated trend and $\nu=4 \times 4 \times 1$ is the volume of a grid cell on one month. In order to satisfy the hardcore condition in (17), we remove dummy points at spatial and temporal distances respectively less than $h_{s}$ and $h_{t}$. Estimated regular parameters are provided in Table 4. 


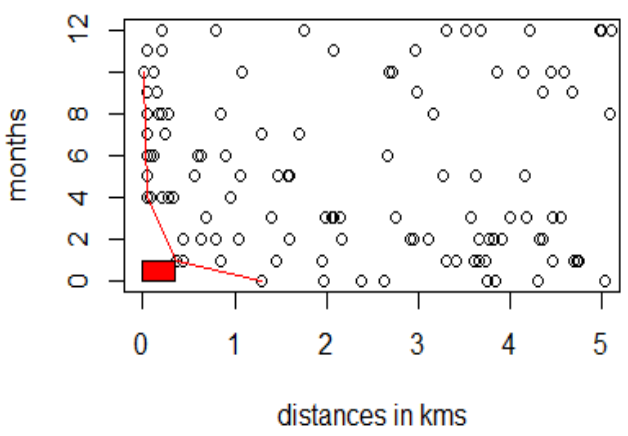

Fig. 4 Spatial and temporal interpoint distances respectively lower than $5 \mathrm{kms}$ and 12 months (black circles). The red line corresponds to the Pareto front and the red rectangle to the hardcore domain.

Table 4 Parameter estimates for $m=6$.

\begin{tabular}{|c|c|c|c|c|c|c|}
\hline \multicolumn{7}{|c|}{ Irregular parameters } \\
\hline$r$ & 0.5 & 1 & 1.5 & 6 & 15 & 20 \\
\hline$q$ & 2 & 4 & 6 & 8 & 12 & 15 \\
\hline \multicolumn{7}{|c|}{ Estimated regular parameters } \\
\hline & $\hat{\gamma}_{1}=2.56$ & $\hat{\gamma}_{2}=2.24$ & $\hat{\gamma}_{3}=4.65$ & $\hat{\gamma}_{4}=0.88$ & $\hat{\gamma}_{5}=1.17$ & $\hat{\gamma}_{5}=0.81$ \\
\hline
\end{tabular}

\subsection{Goodness-of-fit}

The goodness-of-fit is accomplished by simulating point patterns from the fitted model. The first diagnostic can be formulated by summary statistics of point processes. As the second-order characteristics carry most of the information on the spatio-temporal structure (Stoyan, 1992 ; Gonzalez et al., 2016), we only consider the pair correlation function ( $g$-function).

We generate $n_{\text {sim }}=99$ simulations from the fitted hybrid Strauss hardcore model and compute the corresponding second-order summary statistics $g_{i}(u, v), i=1, \ldots, n_{\text {sim }}$, for fixed spatio-temporal distances $(u, v)$. We then build upper and lower envelopes:

$$
U(u, v)=\max _{1 \leq i \leq n_{\text {sim }}} g_{i}(u, v), \quad L(u, v)=\min _{1 \leq i \leq n_{\text {sim }}} g_{i}(u, v),
$$

and compare the summary statistics obtained from the data, $g_{o b s}(u, v)$, to the pointwise envelopes. If it lies outside the envelopes at some spatio-temporal distances $(u, v)$, then we reject at these distances the hypothesis that our data come from our fitted model. Figure 5 shows the spatio-temporal inhomogeneous $g$-function computed on our dataset (blue) and the envelopes obtained from the fitted model (light grey); $g_{o b s}(u, v)$ lies inside the envelopes for all 
$(u, v)$, meaning that the hybrid Strauss hardcore model is suitable for the data.

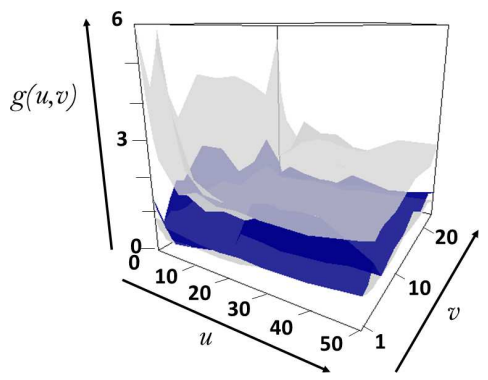

Fig. 5 Envelopes of the spatio-temporal inhomogeneous $g$-function obtained from simulations of the fitted spatio-temporal hybrid Strauss hardcore point process (light grey). The blue surface corresponds to $g_{o b s}$. Temporal separations $v$ are in month and spatial distances $u$ are in kilometer.

In addition, we compute global envelopes and $p$-value of the spatio-temporal $g$-functions based on the Extreme Rank Length (ERL) measure defined in Myllymäki et al. (2017) and implemented in the R package GET (Myllymäki and Mrkvička, 2020). For each point pattern, we consider the long vector $T_{i}$, $i=1, \ldots, n_{\text {sim }}$ (resp. $\left.T_{\text {obs }}\right)$ merging the $g_{i}(\cdot, v)$ (resp. $\left.g_{\text {obs }}(\cdot, v)\right)$ estimates for all considered values $h_{t}$. The ERL measure of vector $T_{i}$ (resp. $T_{o b s}$ ) of length $n_{s t}$ is defined as

$$
E_{i}=\frac{1}{n_{n s}} \sum_{j=1}^{n_{s t}} \mathbb{1}\left\{R_{j} \prec R_{i}\right\},
$$

where $R_{i}$ is the vector of pointwise ordered ranks and $\prec$ is an ordering operator (Myllymäki et al., 2017; Myllymäki and Mrkvička, 2020). The final p-value is obtained by

$$
p_{\text {erl }}=\frac{1+\sum_{i=1}^{n_{\text {sim }}} \mathbb{1}\left\{E_{i} \geq E_{\text {obs }}\right\}}{n_{\text {sim }}+1} .
$$

Due to the global $p$-value $p_{\text {erl }}=0.59$ and the absence of significant regions, that corresponds here to pairs of spatial and temporal distances where the statistics is significantly above or below the envelopes (see Figure 6 and GET package), we conclude that our hybrid Strauss hardcore model can not be rejected a significance level of $1 \%$.

\section{Conclusion}

In this paper, we introduced the spatio-temporal Strauss hardcore point process. The Strauss hardcore model is a Gibbs model for which points are pushed to be at a hardcore distance apart and repel up to a interaction distance which is larger than the hardcore distance. As in Raeisi et al. (2021), inference and 


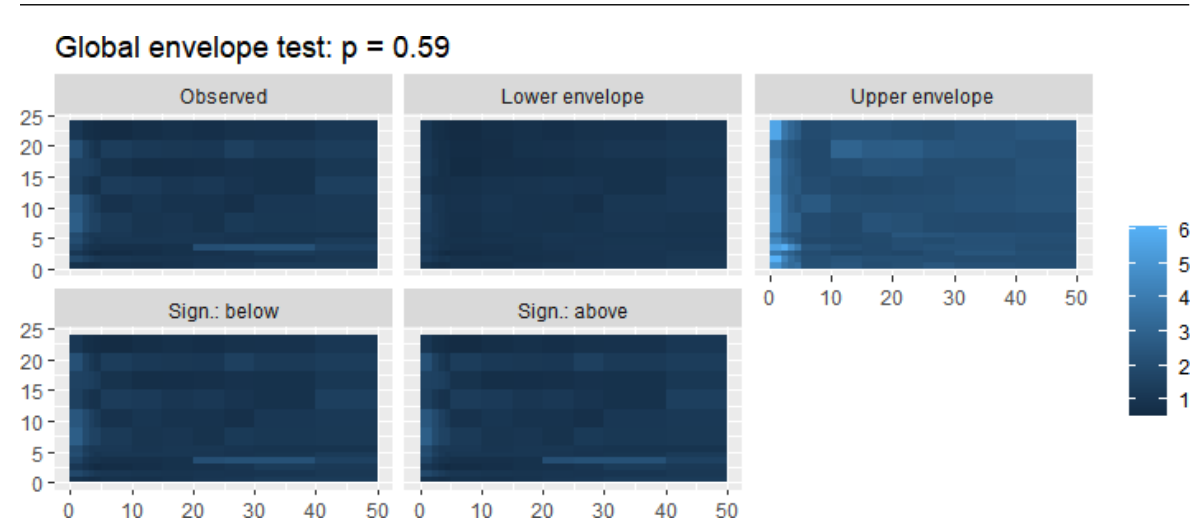

Fig. 6 Top: estimated pair correlation function $\hat{g}_{o b s}$, lower $E_{L}$ and upper $E_{U}$ bounds of the $99 \%$ global rank envelope (ERL). Bottom: differences $E_{o b s}-E_{L}$ and $E_{U}-E_{o b s}$. Negative values (if any) are represented in red and lead to reject the fitted model. Values on the horizontal axis are in kilometers and those on the vertical axis are in months.

simulation of the model were performed with logistic likelihood and birthdeath Metropolis-Hasting algorithm, respectively. A multi-scale version of the model was introduced and applied to wildfires to take into account structural complexity of forest fire occurrences in space and time. We based our model validation on both pointwise and global envelopes and $p$-value based on the Extreme Rank Length (ERL) measure of the spatio-temporal inhomogeneous pair correlation function. Our model could be suitable in other environmental and ecological frameworks, when we want to deal with the complexity of mechanisms governing attraction and repulsion of entities (particles, cells, plants....).

In spatio-temporal Gibbs point process models, the heterogeneity can be captured by estimating a non-constant trend. This spatio-temporal trend is typically considered as a function of covariates by estimating fixed effects in a generalized linear model as we carried out it in this paper and also in Iftimi et al. (2018) and Raeisi et al. (2021). A different approach consists in considering Gibbs models with both random and fixed effects (e.g. see Illian and Hendrichsen, 2010) to take into account complex patterns of spatiotemporal heterogeneity. Vihrs et al. (2020) proposed a new modeling approach for this case and embedded spatially structured Gaussian random effects in trend function of a pairwise interaction process. They introduced the spatial log-Gaussian Cox Strauss point process to capture both structures; aggregation in small-scale and repulsion in large-scale. Rather than spatial pairwise interaction processes in single-scale, we now focus on models derived from the multi-scale classes of combinations of Gibbs and log-Gaussian Cox point processes in space and time, to which we refer to as Cox-Gibbs models. We propose to embed spatio-temporally structured Gaussian random effects in the Gibbs trend function. Due to the hierarchical structure of such models, we can 
formulate and estimate them within a Bayesian hierarchical framework, using the INLA approach.

\section{References}

1. Andersen IT, Hahn U (2016) Matérn thinned Cox processes. Spatial Statistics 15:1-21.

2. Baddeley A, Coeurjolly JF, Rubak E, Waagepetersen R (2014) Logistic regression for spatial Gibbs point processes. Biometrika 101(2):377-392.

3. Baddeley A, Rubak E, Turner R (2015) Spatial Point Patterns: Methodology and Applications with R. Chapman and Hall/CRC Press, London.

4. Baddeley A, Turner R (2000) Practical maximum pseudolikelihood for spatial point patterns (with discussion). Australian and New Zealand Journal of Statistics 42:283-322.

5. Baddeley A, Turner R, Mateu J, Bevan A (2013) Hybrids of Gibbs point process models and their implementation. Journal of Statistical Software 55(11):1-43.

6. Baddeley A, Rubak E, Turner R (2019) Leverage and influence diagnostics for Gibbs spatial point processes. Spatial Statistics 29:15-48.

7. Badreldin N, Uria-Diez J, Mateu J, Youssef A, Stal C, El-Bana M, Magdy A, Goossens R (2015) A spatial pattern analysis of the halophytic species distribution in an arid coastal environment. Environmental Monitoring and Assessment 187: 1-15.

8. Cronie O, van Lieshout M (2015) A J-function for inhomogeneous spatio-temporal point processes. Scandinavian Journal Statistics 42(2):562-579.

9. Das I, Stein A (2016) Application of the Multitype Strauss Point Model for Characterizing the Spatial Distribution of Landslides. Mathematical Problems in Engineering. http:// dx.doi.org/10.1155/2016/1612901.

10. Dereudre D (2019) Introduction to the theory of Gibbs point processes.In: Coupier D (ed) Stochastic Geometry. Lecture Notes in Mathematics vol 2237, Springer, Cham, pp $181-229$.

11. Dereudre D, Lavancier F (2017) Consistency of likelihood estimation for Gibbs point processes. Annals of Statistics 45(2):744-770.

12. Ehrgott M (2005) Multicriteria Optimization. Springer Verlag, Berlin.

13. Juan P (2020) Spatio-temporal hierarchical Bayesian analysis of wildfires with Stochastic Partial Differential Equations. A case study from Valencian Community (Spain). Journal of Applied Statistics 47(5): 927-946. https://doi.org/10.1080/02664763.2019. 1661360 .

14. Juan P, Mateu J, Díaz-Avalos C (2010) Characterizing spatial-temporal forest fire Patterns. In METMA V: International Workshop on Spatio-Temporal Modelling. Santiago de Compostela, Spain.

15. Hurtut T, Landes PE, Thollot J, Gousseau Y, Drouilhet R, Coeurjolly JF (2009) Appearance-guided synthesis of element arrangements by example, Proceedings SIGGRAPH Symposium on Non-Photorealistic Animation and Rendering (NPAR), 51-60.

16. Illian JB, Hendrichsen DK (2010) Gibbs Point Process Models with Mixed Effects. Environmetrics 21:341-353.

17. Illian J, Penttinen A, Stoyan H, Stoyan D (2008) Statistical Analysis and Modelling of Spatial Point Patterns, John Wiley \& Sons, Chichester.

18. Iftimi A, van Lieshout MC, Montes F (2018) A multi-scale area-interaction model for spatio-temporal point patterns. Spatial Statistics 26:38-55.

19. Gabriel E, Opitz T, Bonneu F (2017) Detecting and modeling multi-scale spacetime structures: the case of wildfire occurrences. Journal of the French Statistical Society158(3):86-105.

20. Glass L, Tobler WR (1971) Uniform distribution of objects in a homogeneous field: Cities on a plain. Nature 233:67-68.

21. Gomez-Rubio V (2020) Bayesian Inference with INLA. Chapman \& Hall-CRC, Boca Raton.

22. Gonzalez JA, Rodriguez-Cortes FJ, Cronie O, Mateu J (2016) Spatio-temporal point process statistics: A review. Spatial Statistics 18:505-544. 
23. Matérn B (1960) Spatial variation. Stochastic models and their application to some problems in forest surveys and other sampling investigations. Medd. Statens Skogsforskningsinst 49(5):1-144.

24. Matérn B (1986) Spatial Variation. In: Lecture Notes in Statistics, vol 36. Springer, New York.

25. Mattfeldt T, Eckel S, Fleischer F, Schmidt V (2006) Statistical analysis of reduced pair correlation functions of capillaries in the prostate gland. Journal of Microscopy 223(2):107-119.

26. Mattfeldt T, Eckel S, Fleischer F, Schmidt V (2007) Statistical modelling of the geometry of planar sections of prostatic capillaries on the basis of stationary Strauss hard-core processes. Journal of Microscopy 228:272-281.

27. Mattfeldt T, Eckel S, Fleischer F, Schmidt V (2009) Statistical analysis of labelling patterns of mammary carcinoma cell nuclei on histological sections. Journal of Microscopy $235: 106-118$.

28. Møller J, Diaz-Avalos C (2010). Structured spatio-temporal shot-noise cox point process models, with a view to modelling forest fires. Scandinavian Journal of Statistics 37(1):2-25. https: //www. jstor.org/stable/41000913

29. Møller J, Waagepetersen R P (2004) Statistical Inference and Simulation for Spatial Point Processes. Chapman and Hall/CRC, Boca Raton.

30. Myllymäki M, Kuronen M, Mrkvička T (2021) Testing global and local dependence of point patterns on covariates in parametric models. Spatial Statistics 42:100436.

31. Myllymäki M, Mrkvička T (2020) GET: Global envelopes in R. submitted to Journal of Statistical Software. http://arxiv.org/abs/1911.06583

32. Myllymäki M, Mrkvička T, Grabarnik P, Seijo H, Hahn U (2017) Global Envelope Tests for Spatial Processes. Journal of the Royal Statistical Society: Series B 79:381-404. doi: $10.1111 /$ rssb.12172.

33. Najafabadi ATP, Gorgani F, Najafabadi MO (2015) Modeling forest fires in Mazandaran Province, Iran. Journal of Forestry Research 26:851-858. https://doi.org/10. 1007/s11676-015-0107-z

34. Ogata Y, Tanemura M (1981) Estimation of interaction potentials of spatial point patterns through the maximum likelihood procedure. Annals of the Institute of Statistical Mathematics 33:315-338.

35. Opitz T, Bonneu F, Gabriel E (2020) Point-process based Bayesian modeling of spacetime structures of forest fire occurrences in Mediterranean France. Spatial Statistics 40:100429. https://doi.org/10.1016/j.spasta.2020.100429

36. Papangelou F (1974) The conditional intensity of general point processes and an application to line processes. Probability Theory and Related Fields 28(3):207-226.

37. Pereira P, Turkman K, Amaral-Turkman M, Sa A, Pereira J (2013) Quantification of annual wildfire risk; a spatio-temporal point process approach. Statistica 73(1):55-68. https://doi.org/10.6092/issn.1973-2201/3985

38. Pimont F, Fargeon H, Opitz T, Ruffault J, Barbero R, Martin-StPaul N, Rigolot E, Riviere M, Dupuy J-L (2021) Prediction of regional wildfire activity in the probabilistic Bayesian framework of firelihood, Ecological Applications. doi:10.1002/eap.2316.

39. R Core Team (2016) R: A Language and Environment for Statistical Computing. R Foundation for Statistical Computing, Vienna, Austria, https://www.R-project.org/.

40. Raeisi M, Bonneu F, Gabriel E (2019) On spatial and spatio-temporal multi-structure point process models. Annales de l'Institut de Statistique de l'Université de Paris 63(23):97-114.

41. Raeisi M, Bonneu F, Gabriel E (2021) A spatio-temporal multi-scale model for Geyer saturation point process: application to forest fire occurrences. Spatial Statistics 41: 100492.

42. Ripley BD (1988) Statistical Inference for Spatial Processes. Cambridge University Press.

43. Ripley BD, Kelly FP (1977). Markov point processes. Journal of the London Mathematical Society 15(1):188-192. https://doi.org/10.1112/jlms/s2-15.1.188

44. Rue H, Martino S, Chopin N (2009) Approximate Bayesian inference for latent Gaussian models using integrated nested Laplace approximations (with discussion). Journal of the Royal Statistical Society: Series B (Statistical Methodology) 71(2):319-92. doi:10.1111/j.1467-9868.2008.00700.x. 
45. Serra L, Saez M, Mateu J, Varga D, Juan P, Diaz-Avalos C, Rue H (2014a) Spatiotemporal log-Gaussian Cox processes for modelling wildfire occurrence: the case of Catalonia, 1994-2008. Environmental and Ecological Statistics 21(3):531-563. https://doi. org/10.1007/s10651-013-0267-y

46. Serra L, Saez M, Varga D, Tobías A, Juan P, Mateu J (2012) Spatio-temporal Modelling Of Wildfires In Catalonia, Spain, 1994-2008, Through Log Gaussian Cox Processes. WIT Transactions on Ecology and the Environment 158:39-49. https://doi.org/10. 2495/FIVA120041

47. Serra L, Saez M, Juan P, Varga D, Mateu J (2014b) A spatio-temporal Poisson hurdle point process to model forest fires. Stochastic Environmental Research and Risk Assessment 28(7):1671-1684. https://doi.org/10.1007/s00477-013-0823-x

48. Siino M, Adelfio G, Mateu J, Chiodi M, D'Alessandro A (2017) Spatial pattern analysis using hybrid models: an application to the Hellenic seismicity. Stochastic Environmental Research and Risk Assessment 31(7):1633-1648.

49. Siino M, D'Alessandro A, Adelfio G, Scudero S, Chiodi M (2018) Multiscale processes to describe the eastern sicily seismic sequences. Annals of Geophysics 61(2).

50. Stoyan D (1992) Statistical estimation of model parameters of planar Neyman-Scott cluster processes. Metrika 39(1):67-74. https://doi.org/10.1007/BF02613983

51. Stoyan D (2016) Point process statistics: application to modern and contemporary art and design. Journal of Mathematics and the Arts 10:20-34

52. Sweeney S, Gómez-Antonio M (2016) Localization and industry clustering econometrics: An assessment of Gibbs models for spatial point processes. Journal of Regional Science 56:257-287.

53. Taylor DB, Dhillon HS, Novlan TD, Andrews J G (2012) Pairwise interaction processes for modeling cellular network topology. Proceedings IEEE GLOBECOM in Anaheim.

54. Teichmann J, Ballani F, Boogaart KG van den (2013) Generalizations of Matérn's hardcore point processes. Spatial Statistics 9:33-53.

55. Turner R (2009) Point patterns of forest fire locations. Environmental and Ecological Statistics 16:197-223. https://doi.org/10.1007/s10651-007-0085-1

56. van Lieshout MNM (2000) Markov Point Processes and Their Applications. Imperial College Press, London.

57. Vihrs N, Møller J, Gelfand AE (2021) Approximate Bayesian inference for a spatial point process model exhibiting regularity and random aggregation, Scandinavian Journal of Statistics: Theory and Applications. https://doi.org/10.1111/sjos.12509

58. Wang X, Zheng G, Yun Z, Moskal LM (2020). Characterizing tree spatial distribution patterns using discrete Aerial Lidar Data. Remote Sensing 12(4): 712. https://doi.org/ 10.3390/rs12040712

59. Ying Q, Zhao Z, Zhou Y, Li R, Zhou X, Zhang H (2014). Characterizing spatial patterns of base stations in cellular networks. Proceedings IEEE/CIC International Conference on communications in China (ICCC), 490-495. 